\title{
The effect of topical finasteride in treatment of idiopathic hirsutism
}

Yahia Ibrahim Yahia*1, Nisreen J. Mohammed ${ }^{1}$, Wisam A. Ameen ${ }^{2}$

\begin{abstract}
Hirsutism relatively is a common medical problem and an important influence on about $5-10 \%$ of women of reproductive age. Fifty five female shares in the study, seventeen of them did not continue the study, therefore we evaluated 38 females, aged 18-52 years old. In our study we compared pre-treatment and post-treatment for the color of the hairs and the hair removal frequency and the pain during hair removal for four groups which are good response group and moderate response group and mild response group and no response group, by subjective evaluation of all the patients noted a diminished rate of hair growth (fewer times needed for shaving) and a decrement in the density of hairs on the chin area and change in color of the hair but there was no significant difference between groups regarding hair color and there were highly significant difference in hair removal frequency before and after treatment $(\mathrm{P}<0.001)$ when we used finasteride cream $1 \%$ for 3 months. Six months of topically applied finasteride $(0.5 \%)$ does not effect on number and thickness of facial hirsutism significantly. Despite lack of objective changes, on questioning, most patients in finastride group perceived a decrease in hair growth with time.
\end{abstract}

Keywords: Hirsutism; 5- $\alpha$-dihydrotestosterone; TSH; FH; LH

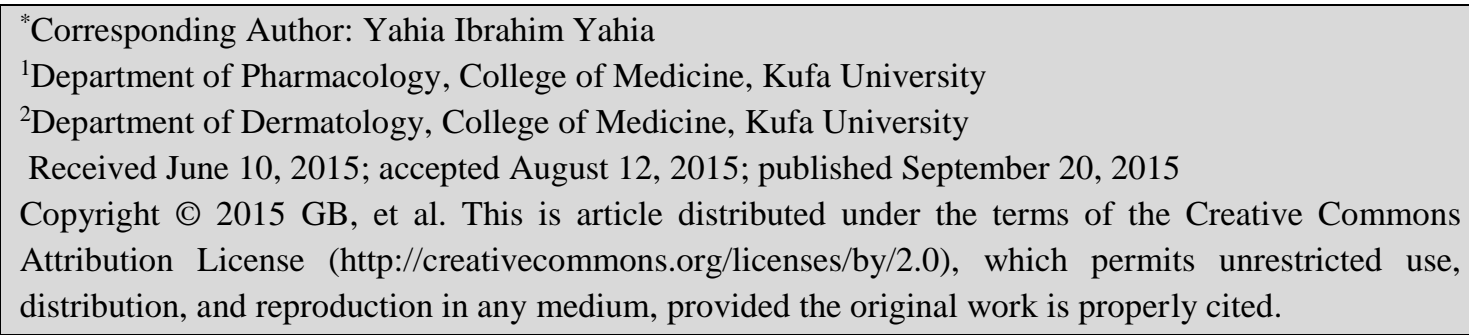

\section{Introduction}

The term hirsutism defines as presence of coarser, thicker and terminal hair in women in a male like pattern and locations [1]. Hirsutism relatively is a common medical problem and an important influence on about $5-10 \%$ of women of reproductive age $[2,3]$. The prevalence of hirsutism is dependent on the ethnic and racial origin of the population under study, an incidence of 
hirsutism of $8 \%$ was found in the US [4].

The androgen responsible for the change in voice and the increase in muscle mass in women is testosterone, and that responsible for hirsutism is $5-\alpha-$

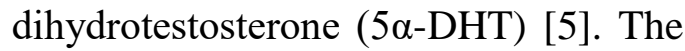
testosterone is the key circulating androgen which is produced by the ovaries and adrenals either as testosterone or as prohormones (mainly androstenedione and dehydroepiandrosteron sulfate) which are metabolized into testosterone in the peripheral tissues such as fat [6]. Testosterone is converted into dihydrotestosterone (DHT) in the peripheral tissue by the enzyme 5- $\alpha$ reductase. DHT is the most potent androgen in the body. The phenomena of increased conversion rate of DHT in the target area may help to clarify the increased sensitivity of hair follicles to androgens [3]. "Idiopathic hirsutism" (IH), also called simple or peripheral hirsutism, while IH is often referred to as "familial hirsutism," this represents a misnomer. In fact, it is now well established that other forms of hyperandrogenism, e.g., PCOS and NCAH, also demonstrate a strong familial predisposition [7].

\section{Material and Method}

The present study was carried out in Collage of Medicine Babylon University from November 2013 - November 2014 for treatment Fifty five females were enrolled in this study. Their age was between (18-55 years) and the mean of their age was 32.26. Those females were chosen from consulting dermatologist department in Mergan medical city in Babylon. These patients complained from hirsutism and given Finasteride $1 \%$ cream and was measured $\mathrm{TSH}$, FSH, LH and free Testosterone pretreatment and post-treatment and also measured the:

- Hair color

- Hair removal frequency of the patients

- Pain severity before and after treatment

- F-Gallawy score

- Visual analogue score

The follow up continue every 15 day and after 3 months (end of study) it was measured the same parameters. The patients were asked about the name, age, marital state, parity, blood group, onset of hirsutism, duration, distribution of hirsutism as it occur in the face only or other body area, hair type as dark hair or fine hair, other features of hyperandrogenism as acne and hair loss and disturbance of the menstrual cycle. 
Previous treatment as some drugs may case or exacerbate hirsutism, previous hair removal, number of hair removal, and history of contraceptive, history of other disease.

Follow up with each patient every two weeks and evaluate the case by evaluating the number of the hair and the roughness of the hair by Ferriman Galway Scoring Sheet and we know the improvement the patient through this score. All the patients gave informed consent for their participation in our study after reading the protocol of this experiment. They had not used any other therapy for idiopathic hirsutism for at least the six previous months. They were told that finasteride could affect a male fetus and consequently pregnancy was contraindicated during the treatment and so effective contraceptive must be used. They were also informed that potential side effects of finasteride were unknown in women and they should report any possible side effects during the medication. The patients were explained not to use any other drug for $\mathrm{IH}$ at the same time.

Moreover electrolysis, waxing and plucking were not permitted during the treatment whereas shaving was permitted for subjective evaluation of hair growth by patients. The degree of hirsutism in the chin area was determined by Ferrriman-Gallawey score. The scale is form 0 (absence of terminal hairs) to 4 (extensive terminal hair growth). Then they received finasteride cream $1 \%$ on their chins once a day for 3 months.

They were explained to clean the chin area before usage and to avoid using powder, lotions, and sprays two hours after cream. The finasteride cream made as the Pure finasteride was procured from Samarra a drug industry, firstly 1 gram Finasteride powder weighted and putted beaker and dissolve it in $3 \mathrm{ml}$ of alcohol and shaking it for 4 minutes until it dissolved completely and became clear after that we complete the weight to 100 gram with aquasoft cream (Ajanta company) and shake the combination for 5 minutes by spatula and then weight the finasteride cream in small plastic containers each one containing 10 grams from finasteride cream to be used by the patients.

Then the patients allocated to four groups according the visual analogue scale and the F.G score:

Group (1): good response group

Group (2): moderate response group

Group (3): mild response group

Group (4): non response group

The definition of each one: 
1. Good response group: are the patients whom have response to the treatment more than $>75 \%$.

2. Group (2): moderate response group: are the patients whom have response to the treatment $50-75 \%$

3. Group (3): Mild response group: are the patients whom have response to the treatment 25 $50 \%$.

4. No response group: are the patients whom have response to the treatment less than $<25 \%$.

The patients were seen in consultation at 3 months intervals. Questions were asked about the side effects, menstrual abnormalities and also patient's selfevaluation of the clinical effects of the treatment.

After six months, the mean Caliber of three plucked hairs and the FerrimanGallwey score of the chin area was assessed again. Data are presented as mean \pm SD or percentage. Statistical analyses were performed using SPSS software version 20:0:0 and paired TTest for comparison of quantitative variables was used to compare the hair caliber before and after medication. $\mathrm{P}$ values less than 0.05 were statistically significant.

\section{Results}

Statistical analysis

Fifty five females shares in the study. Seventeen of them did not continue the study, therefore we evaluated 38 females, aged 18-52 years old. Data are presented as mean $\pm \mathrm{SD}$.

Means of TSH ,FH, LH and Free testosterone for two groups were compared with paired student's t-test in SPSS software by using SPSS (statistical package for social sciences) version 20 in which we use paired T-test, chi square test, wilcoxon test, Freidman test according to the need.

We set $\mathrm{P}$ value $<0.05$ as significant. A Pvalue less than 0.05 was considered statistically significant. Out of fifty five participants, seventeen were excluded because they stopped the medication. The thirty eight remained patients with the mean age was $32.2 \pm 8.04$ continued the medication throughout the 3-month study period.

None of the females reported any systemic problems with irregularity of menstrual periods, nausea, vomiting, diarrhea, abdominal pain or headache. Acne was the only problem reported in 5 patients $(7.6 \%)$ on the chin area where the drug was applied. By subjective evaluation all the patients noted a diminished rate of hair growth (fewer 
times needed for shaving) and a decrement in the density of the hairs on the chin area. By objective evaluation, free testosterone was decreased after medication that was statistically significant $\mathrm{P}<0.001$.

The Ferriman-Gallwey score of the chin area statistically decreased from 2.3421 to 1.9737 after a 3- month finasteride cream therapy $(\mathrm{P}<0.001)$ By Pearson correlation test.

\begin{tabular}{|l|r|r|l|}
\hline \multirow{2}{*}{ Variable } & Pretreatment & Post-treatment & \multirow{2}{*}{ P value } \\
\cline { 2 - 3 } & Mean \pm SE & Mean \pm SE & \\
\hline TSH(uIU/ml) & $3.0884 \pm 0.50754$ & $2.2243 \pm 0.29844$ & 0.043 \\
\hline LH(pg/ml) & $9.2077 \pm 1.49261$ & $9.4453 \pm 1.33724$ & 0.779 \\
\hline FSH(mIU/ml) & $7.3907 \pm 1.20073$ & $8.3816 \pm 1.79212$ & 0.266 \\
\hline $\begin{array}{l}\text { Free testosterone } \\
\text { (pg/ml) }\end{array}$ & $2.9387 \pm 0.32011$ & $2.6127 \pm 0.41021$ & 0.179 \\
\hline
\end{tabular}

Table 1.

Hormonal assessment before and after treatment for all patients.

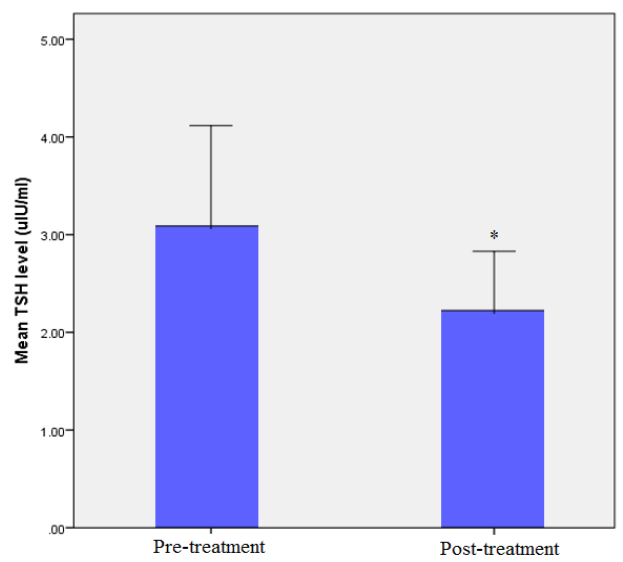

Figure 1.

TSH level before and after treatment for all patients. The TSH level decreased after treatment with topical finasteride cream as some patients also have changes in TSH. There are cases where the Post-Finasteride Syndrome induced hypothyroidism. However, it is not most cases.

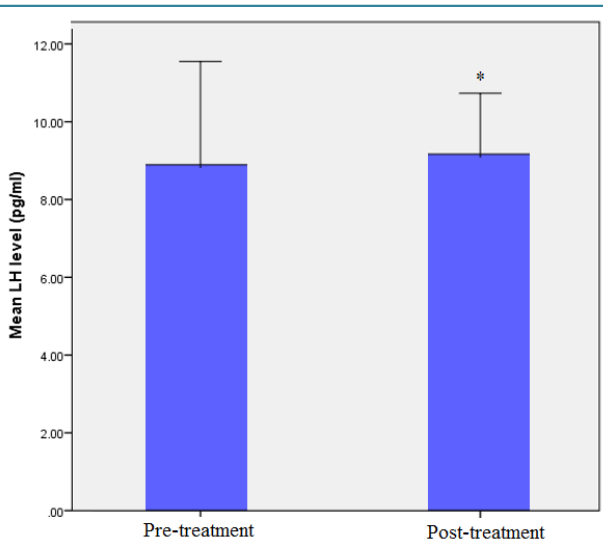

Figure 2.

LH level before and after treatment for all patients

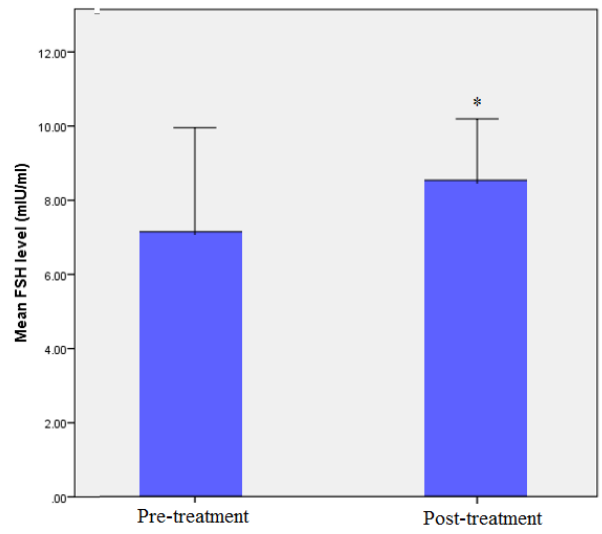

Figure 3.

FSH level before and after treatment for all patients, and demonstrated no significant difference of FSH level after treatment of all the patients.

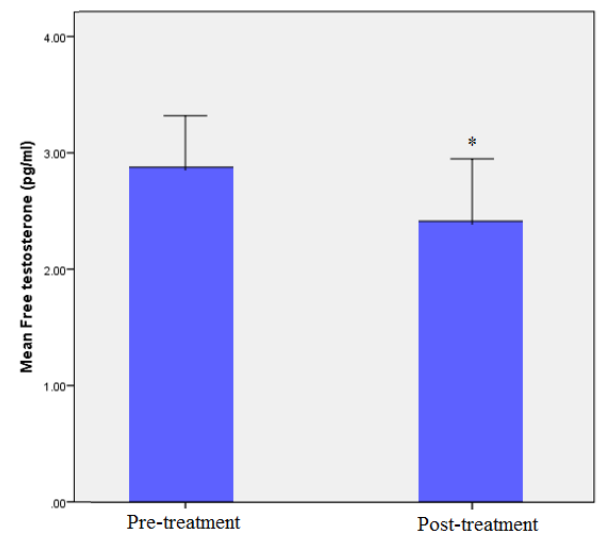

Figure 4.

Free testosterone level before and after treatment, and demonstrated no significant 
American Journal of BioMedicine

AJBM 2015; 3(9):552 - 566

difference in free testosterone level after treatment of all the patients.

\begin{tabular}{|l|l|l|l|}
\hline \multirow{2}{*}{ Variable } & Pretreatment & Post-treatment & \multirow{2}{*}{ P value } \\
\cline { 2 - 3 } & Mean \pm SE & Mean \pm SE & \\
\hline TSH(ulU/ml) & $2.9792 \pm 0.85394$ & $2.4800 \pm 0.60835$ & 0.133 \\
\hline LH(pg/ml) & $6.8577 \pm 1.92950$ & $7.9977 \pm 2.34525$ & 0.310 \\
\hline FSH(mlU/ml) & $7.8346 \pm 2.78310$ & $10.4385 \pm 4.82816$ & 0.253 \\
\hline $\begin{array}{l}\text { Free } \\
\text { testosterone(pg/ml) }\end{array}$ & $2.6823 \pm 0.33680$ & $1.4847 \pm 0.28468$ & 0.006 \\
\hline
\end{tabular}

Table 2.

Hormonal assessment before and after treatment among good response group.

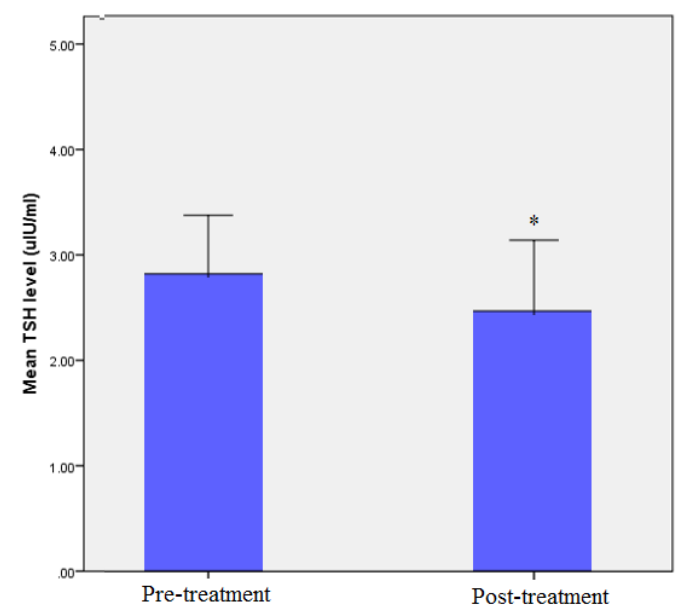

\section{Figure 5.}

TSH level before and after treatment among good response group. The TSH level decreased after treatment with topical finasteride cream as some patients also have changes in TSH.

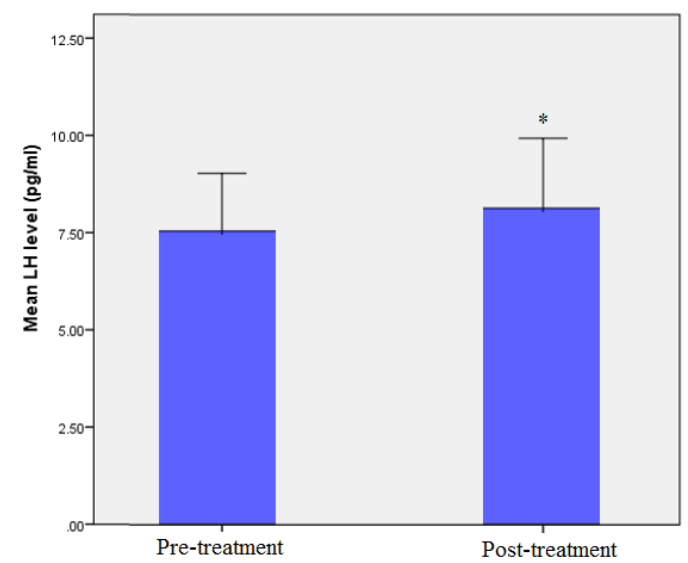

Figure 6.

LH level before and after treatment among good response group, without significant difference of
LH level after treatment for good response group patients.

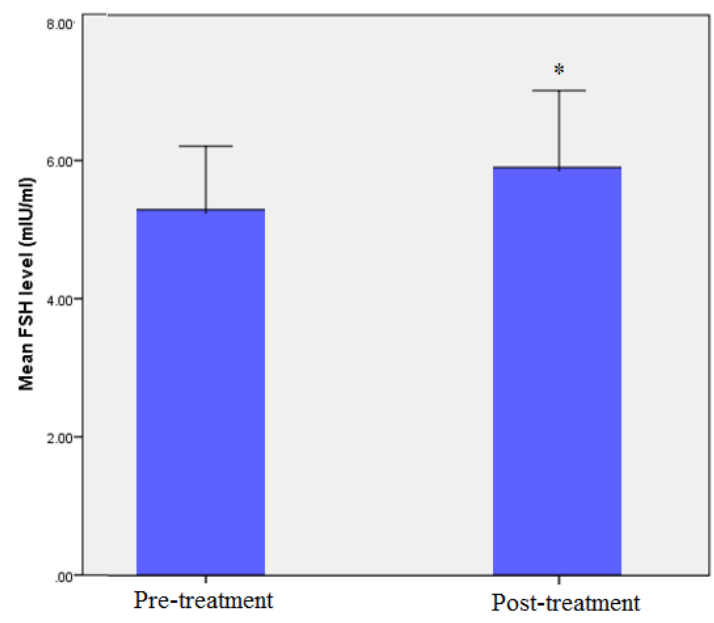

Figure 7.

FSH level before and after treatment among good response group, there is no significant difference of FST level after treatment for good response group patients.

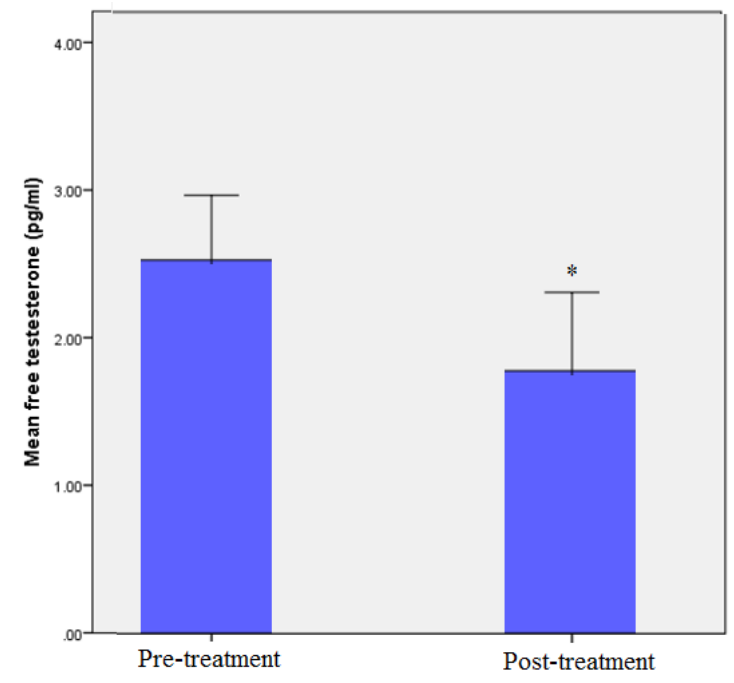

Figure 8.

Free testosterone level before and after treatment among good response group. Finasteride is considered to be a potent non-steroidal antiandrogen that acts by inhibiting the type 2-5 $\alpha$-reductase isoenzyme, blocking the conversion of testosterone to 5- $\alpha$-DHT, with a significant reduction of serum $5 \alpha$-DHT levels and no prominent side-effects. 


\section{American Journal of BioMedicine \\ AJBM 2015; 3(9):552 - 566}

\begin{tabular}{|l|l|l|l|}
\hline \multirow{2}{*}{ Variable } & Pretreatment & Post-treatment & \multirow{2}{*}{ P value } \\
\cline { 2 - 3 } & Mean \pm SE & Mean \pm SE & \\
\hline TSH(ulU/ml) & $2.9479 \pm 0.68128$ & $2.4300 \pm 0.54213$ & 0.094 \\
\hline LH(pg/ml) & $11.4036 \pm 3.11762$ & $9.8929 \pm 2.47450$ & 0.375 \\
\hline FSH(mlU/ml) & $7.9464 \pm 1.95649$ & $7.1471 \pm 1.94245$ & 0.145 \\
\hline $\begin{array}{l}\text { Free } \\
\text { testosterone(pg/ml) }\end{array}$ & $2.4264 \pm 0.54332$ & $2.5743 \pm 0.84006$ & 0.737 \\
\hline & & & \\
\hline
\end{tabular}

Table 3.

Hormonal assessment before and after treatment among moderate response group.

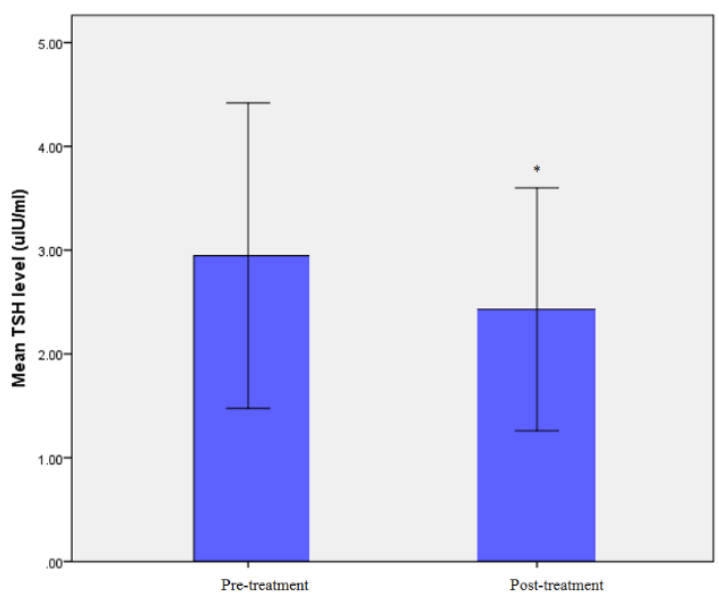

Figure 9.

TSH level before and after treatment among moderate response group, in moderate response group we have slightly decrease in the TSH levels.

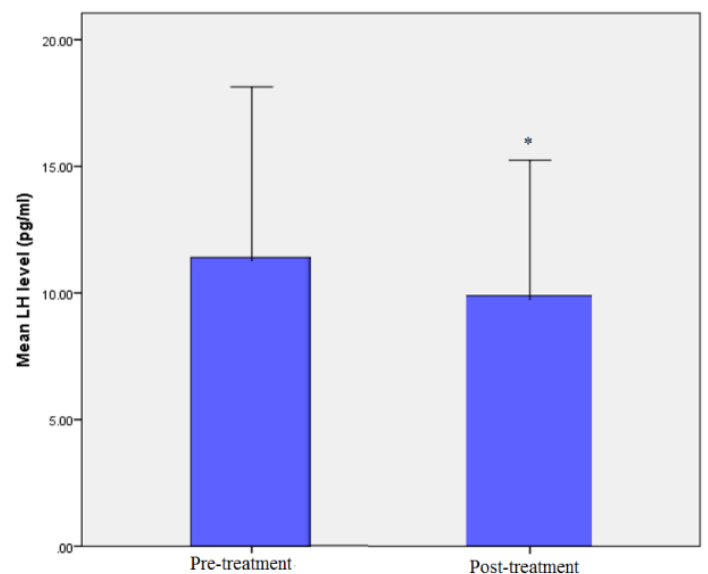

Figure 10.

LH level before and after treatment among moderate response group, in moderate response group we have slightly decrease in the LH levels.

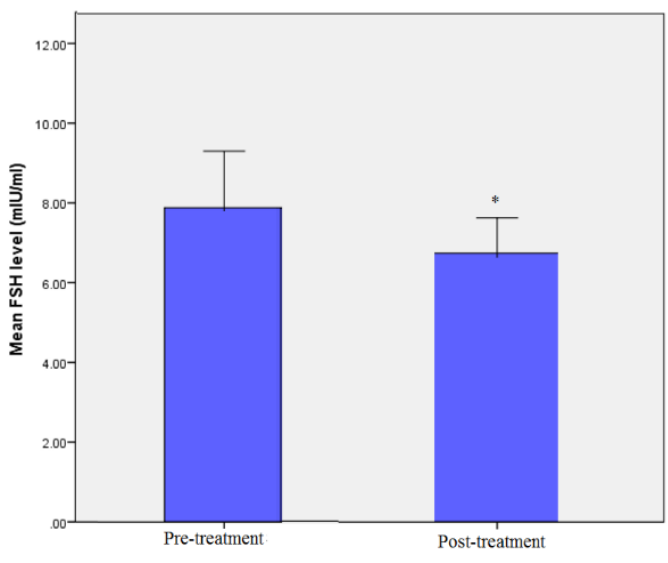

Figure 11.

FSH level before and after treatment among moderate response group, in moderate response group we have slightly increase in the FSH levels.

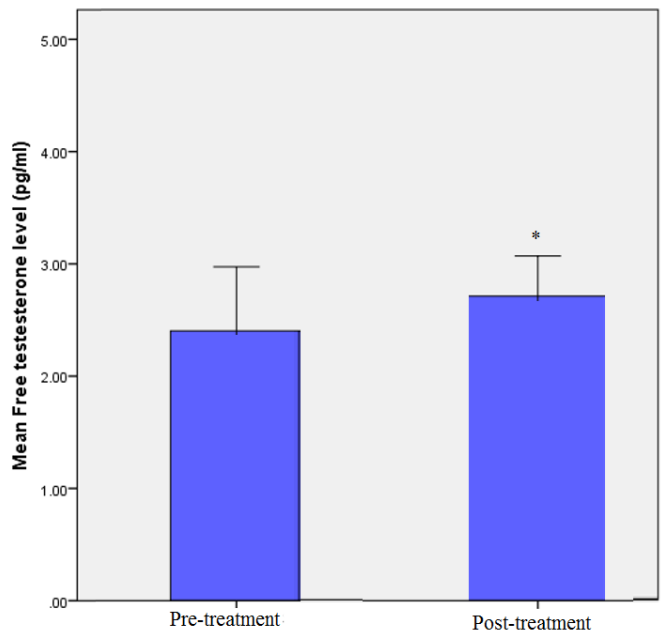

Figure 12.

Free test level before and after treatment among moderate response group, in moderate response group we have slightly increase in the free testosterone levels.

\begin{tabular}{|l|l|l|l|}
\hline \multirow{2}{*}{ Variable } & Pretreatment & Post-treatment & \multirow{2}{*}{ P value } \\
\cline { 2 - 3 } & Mean \pm SE & Mean \pm SE & \\
\hline TSH(ulU/ml) & $2.9479 \pm 0.68128$ & $2.4300 \pm 0.54213$ & 0.094 \\
\hline LH(pg/ml) & $11.4036 \pm 3.11762$ & $9.8929 \pm 2.47450$ & 0.375 \\
\hline FSH(mlU/ml) & $7.9464 \pm 1.95649$ & $7.1471 \pm 1.94245$ & 0.145 \\
\hline $\begin{array}{l}\text { Free } \\
\text { testosterone(pg/ml) }\end{array}$ & $2.4264 \pm 0.54332$ & $2.5743 \pm 0.84006$ & 0.737 \\
\hline
\end{tabular}

Table 4.

Hormonal assessment before and after treatment among mild response group. 


\section{American Journal of BioMedicine \\ AJBM 2015; 3(9):552 - 566}

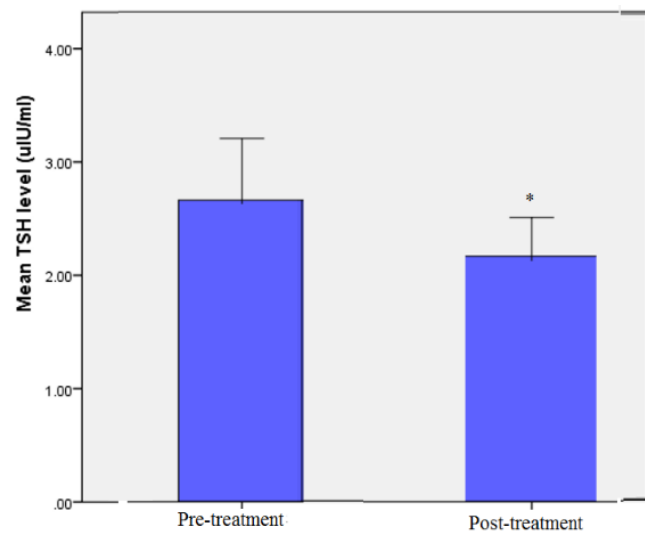

\section{Figure 13.}

TSH level before and after treatment among mild response group, in mild response group we have slightly decrease in the TSH levels.

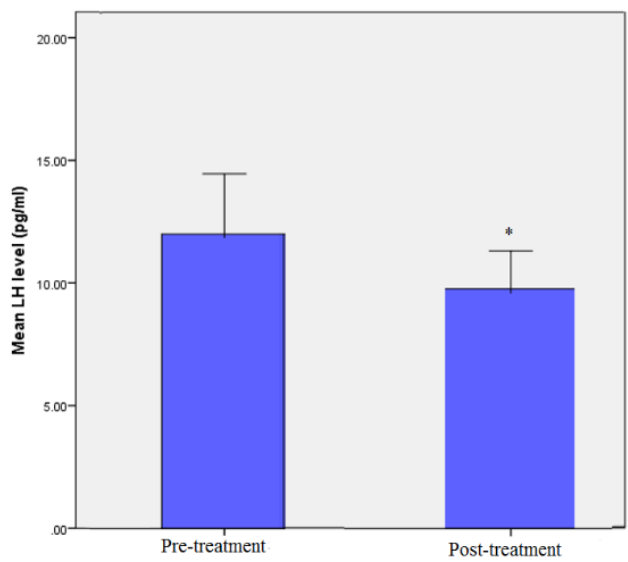

Figure 14.

LH level before and after treatment among mild response group, $\mathrm{n}$ mild response group we have slightly decrease in the LH levels.

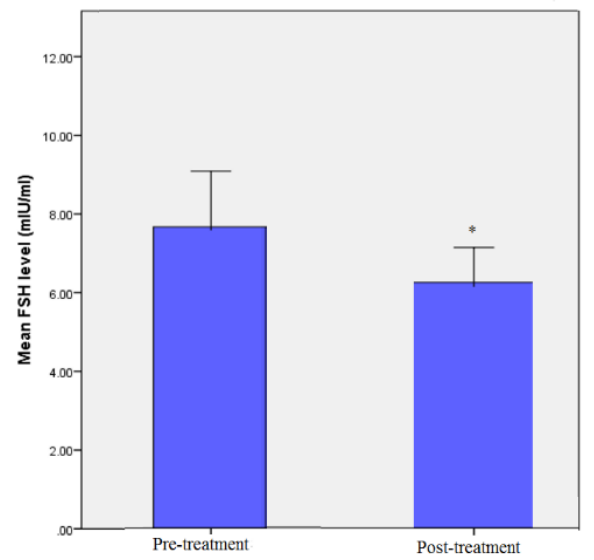

Figure 15.

FSH level before and after treatment among mild response group.

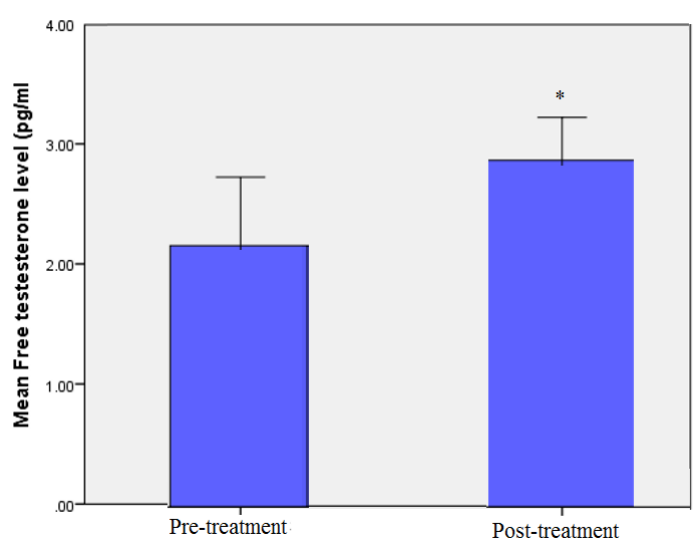

Figure 16.

Free test level before and after treatment among mild response group, in mild response group we have slightly increase in the free testosterone levels.

\begin{tabular}{|l|l|l|l|}
\hline \multicolumn{1}{|c|}{ Variable } & Pretreatment & Post-treatment & \multirow{2}{*}{ P value } \\
\cline { 2 - 3 } & Mean \pm SE & Mean \pm SE & \\
\hline TSH(ulU/ml) & $3.3964 \pm 1.21531$ & $1.6603 \pm 0.28699$ & 0.224 \\
\hline $\mathbf{L H}(\mathbf{p g} / \mathbf{m l})$ & $9.1904 \pm 2.41841$ & $10.5864 \pm 2.12646$ & 0.376 \\
\hline FSH $(\mathbf{m l U} / \mathbf{m l})$ & $6.1587 \pm 0.94639$ & $7.5218 \pm 0.99069$ & 0.357 \\
\hline $\begin{array}{l}\text { Free } \\
\text { testosterone(pg/ml) }\end{array}$ & $3.8936 \pm 0.72674$ & $3.9945 \pm 0.72578$ & 0.746 \\
\hline
\end{tabular}

\section{Table 5.}

Hormonal assessment before and after treatment among no response group.

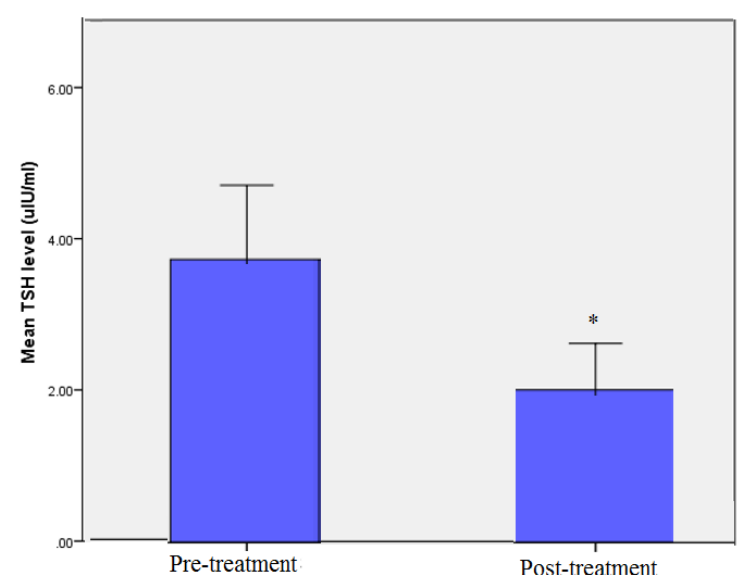

Figure 17.

TSH level before and after treatment among no response group. 


\section{American Journal of BioMedicine \\ AJBM 2015; 3(9):552 - 566}

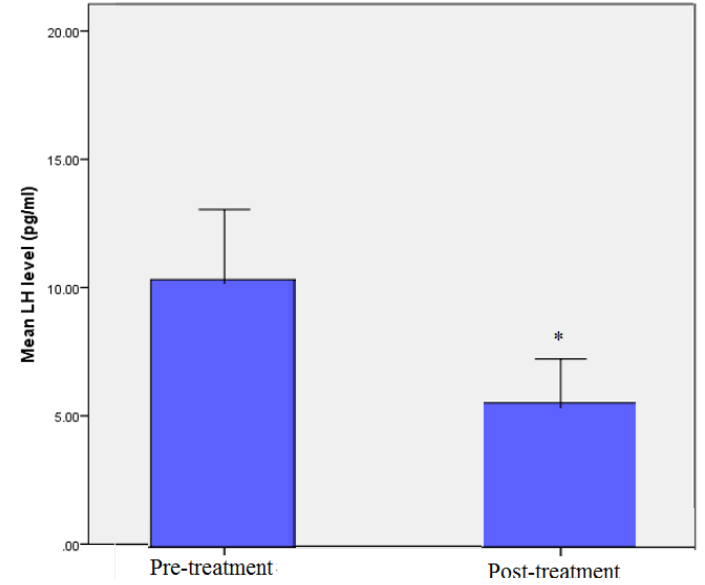

Figure 18.

LH level before and after treatment among no response group.

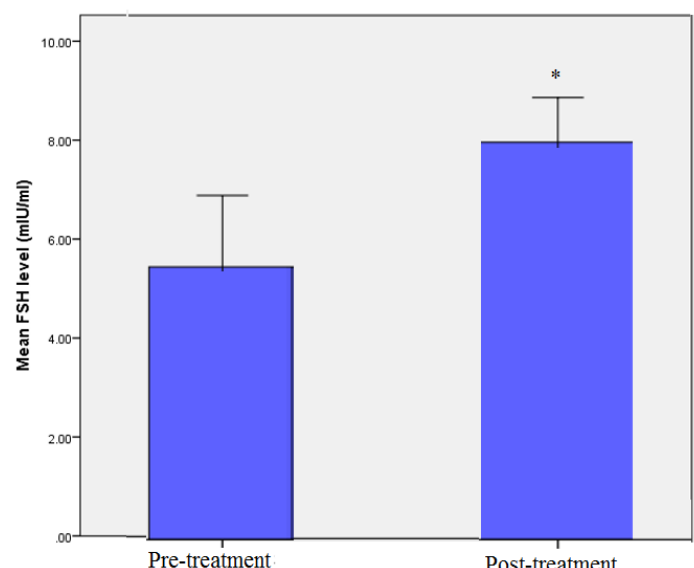

Figure 19.

FSH level before and after treatment among no response group.

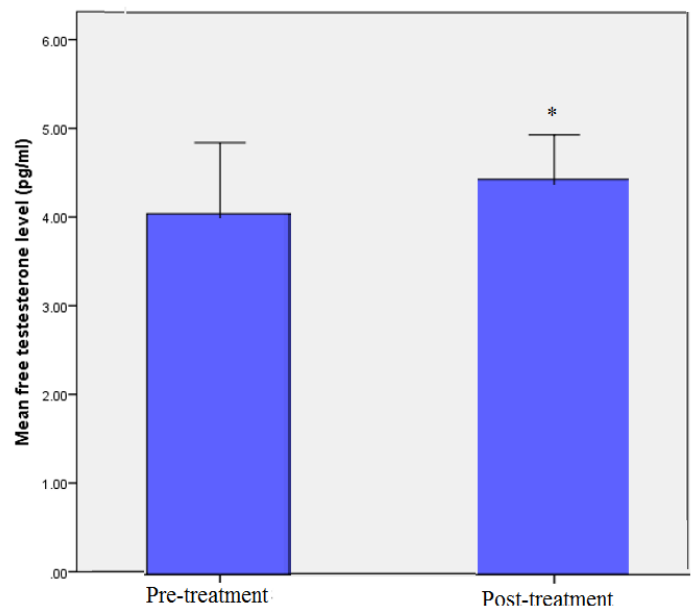

Figure 20.

Free test level before and after treatment among no response group.

\begin{tabular}{|c|c|c|c|c|}
\hline & & \multicolumn{2}{|c|}{ Hair color post } & \multirow{2}{*}{$P$ value } \\
\hline & & dark hair & light hair & \\
\hline \multirow{2}{*}{ No response } & \multirow{2}{*}{ Dark hair (8) } & 4 & 4 & \multirow{4}{*}{0.024} \\
\hline & & $50.0 \%$ & $50.0 \%$ & \\
\hline \multirow{2}{*}{ Response } & \multirow{2}{*}{ Dark hair(30) } & 4 & 26 & \\
\hline & & $13.3 \%$ & $86.7 \%$ & \\
\hline \multirow{2}{*}{\multicolumn{2}{|c|}{ Total }} & 8 & 30 & \\
\hline & & $21.1 \%$ & $78.9 \%$ & \\
\hline
\end{tabular}

Table 6.

Hair color (for all Patients).

\begin{tabular}{|c|c|c|c|c|}
\hline & & \multicolumn{2}{|c|}{ Hair color post } & \multirow[t]{2}{*}{ Total } \\
\hline & & dark hair & light hair & \\
\hline \multirow[t]{2}{*}{ Good response } & \multirow[t]{2}{*}{ Dark hair(13) } & 2 & 11 & 13 \\
\hline & & $15.4 \%$ & $84.6 \%$ & $100.0 \%$ \\
\hline \multirow[t]{2}{*}{ Moderate response } & \multirow[t]{2}{*}{ Dark hair(14) } & 2 & 12 & 14 \\
\hline & & $14.3 \%$ & $85.7 \%$ & $100.0 \%$ \\
\hline \multirow[t]{2}{*}{ Mild response } & \multirow[t]{2}{*}{ Dark hair(3) } & 0 & 3 & 3 \\
\hline & & $0.0 \%$ & $100.0 \%$ & $100.0 \%$ \\
\hline \multirow[t]{2}{*}{ No response } & \multirow[t]{2}{*}{ Dark hair(8) } & 4 & 4 & 8 \\
\hline & & $50.0 \%$ & $50.0 \%$ & $100.0 \%$ \\
\hline \multirow[t]{2}{*}{ Total } & & 8 & 30 & 38 \\
\hline & & $21.1 \%$ & $78.9 \%$ & $100.0 \%$ \\
\hline
\end{tabular}

Table 7.

Hair color for each group

\begin{tabular}{|c|c|c|}
\hline & Mean \pm SD & P value \\
\hline $\begin{array}{c}\text { Hair removal frequency } \\
\text { pretreatment }\end{array}$ & $4.50 \pm 3.311$ & $<0.001$ \\
\cline { 1 - 2 } $\begin{array}{c}\text { Hair removal frequency post- } \\
\text { treatment }\end{array}$ & $2.789 \pm 2.623$ & \\
\hline
\end{tabular}

Table 8.

Hair removal frequency of the patients.

\begin{tabular}{|l|c|c|c|}
\hline & Mean & Std. Deviation & P value \\
\hline Hair removal frequency pre & 2.85 & 0.987 & $<0.001$ \\
\hline Hair removal frequency post & 1.3077 & 0.48038 & \\
\hline
\end{tabular}

Table 9.

Hair removal- good response.

\begin{tabular}{|l|c|c|c|}
\hline & Mean & Std. Deviation & P value \\
\hline Hair removal frequency pre & 2.85 & 0.987 & $<0.001$ \\
\hline Hair removal frequency post & 1.3077 & 0.48038 & \\
\hline
\end{tabular}

Table 10.

Hair removal-moderate response. 


\section{American Journal of BioMedicine AJBM 2015; 3(9):552 - 566}

\begin{tabular}{|l|l|l|l|}
\hline & Mean & Std. Deviation & P value \\
\hline Hair removal frequency pre & 8.00 & 6.928 & 0.251 \\
\hline Hair removal frequency post & 5.3333 & 4.04145 & \\
\hline
\end{tabular}

Table 11.

Hair removal-mild response.

\begin{tabular}{|l|l|l|l|}
\hline & Mean & Std. Deviation & P value \\
\hline Hair removal frequency pre & 7.00 & 4.000 & 0.04 \\
\hline Hair removal frequency post & 5.5000 & 4.47214 & \\
\hline
\end{tabular}

Table 12.

Hair removal-no response.

\begin{tabular}{|c|c|c|c|c|}
\hline & $\begin{array}{l}\text { Sever } \\
\text { Pain }\end{array}$ & $\begin{array}{l}\text { Moderate } \\
\text { pain }\end{array}$ & No pain & $P$ value \\
\hline \multirow{2}{*}{ Good response } & 0 & 6 & 7 & \multirow{8}{*}{0.001} \\
\hline & $0.0 \%$ & $46.2 \%$ & $53.8 \%$ & \\
\hline \multirow{2}{*}{$\begin{array}{l}\text { Moderate } \\
\text { response }\end{array}$} & 1 & 10 & 3 & \\
\hline & $7.1 \%$ & $71.4 \%$ & $21.4 \%$ & \\
\hline \multirow{2}{*}{ Mild response } & 0 & 3 & 0 & \\
\hline & $0.0 \%$ & $100.0 \%$ & $0.0 \%$ & \\
\hline \multirow{2}{*}{ No response } & & 3 & 0 & \\
\hline & & $37.5 \%$ & $0.0 \%$ & \\
\hline \multirow{2}{*}{ Total } & & 22 & 10 & \\
\hline & & $57.9 \%$ & $26.3 \%$ & \\
\hline
\end{tabular}

Table 13.

Pain severity before and after treatment.

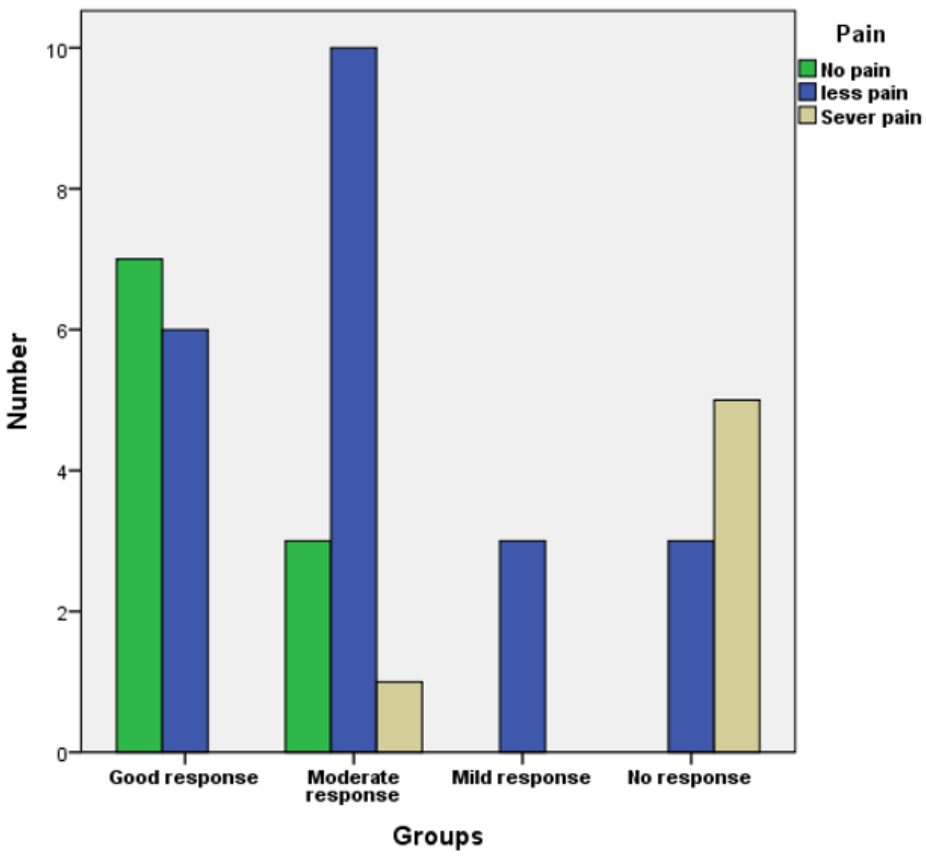

Figure 21.

Pain severity among different groups.

\begin{tabular}{|l|c|c|c|c|}
\hline & Time & Mean & Std. Deviation & P value \\
\hline \multirow{2}{*}{ Pair 1 } & Pretreatment & 3.3158 & 0.66191 & $<0.001$ \\
\cline { 2 - 4 } & 1month & 2.3421 & 0.96636 & \\
\hline \multirow{3}{*}{ Pair 2 } & 1 month & 2.3421 & 0.96636 & \multirow{2}{*}{0.058} \\
\cline { 2 - 4 } & 2month & 2.1316 & 0.93494 & \\
\hline \multirow{2}{*}{ Pair 3 } & 2month & 2.1316 & 0.93494 & \multirow{2}{*}{0.057} \\
\cline { 2 - 4 } & Third month & 1.9737 & 0.91495 & \multirow{2}{*}{$<0.001$} \\
\hline Pair 4 & Pretreatment & 3.3158 & 0.66191 & \\
\cline { 2 - 4 } & Third month & 1.9737 & 0.91495 & \\
\hline
\end{tabular}

Table 14.

Follow up with F-Gallawy score, there is a significant difference in the score with time so the score is higher in the first 2 weeks while it is lower in the sixth 2 weeks, (Friedman test).

\begin{tabular}{|l|c|c|c|c|}
\hline & & Mean & Std. Deviation & P value \\
\hline \multirow{2}{*}{ Pair 1 } & Pretreatment & 3.4615 & 0.51887 & \multirow{2}{*}{$<0.001$} \\
\cline { 2 - 4 } & 1month & 1.7692 & 0.92681 & \\
\hline \multirow{2}{*}{ Pair 2 } & 1 month & 1.7692 & 0.92681 & \multirow{2}{*}{0.502} \\
\cline { 2 - 4 } & 2month & 1.6154 & 0.65044 & \\
\hline \multirow{2}{*}{ Pair 3 } & 2month & 1.6154 & 0.65044 & \multirow{2}{*}{0.337} \\
\cline { 2 - 4 } & Third month & 1.6923 & 0.63043 & \multirow{2}{*}{$<0.001$} \\
\hline \multirow{2}{*}{ Pair 4 } & Pretreatment & 3.4615 & 0.51887 & \\
\cline { 2 - 5 } & Third month & 1.6923 & 0.63043 & \\
\hline
\end{tabular}

Table 15.

F-Gallawy good response, in good response group they had significant differences between pre-treatment and first month and between pretreatment and third month.

\begin{tabular}{|l|c|l|l|l|}
\hline \multicolumn{2}{|c|}{} & Mean & Std. Deviation & P value \\
\hline \multirow{2}{*}{ Pair 1 } & Pretreatment & 3.2143 & 0.69929 & \multirow{2}{*}{0.001} \\
\cline { 2 - 4 } & 1month & 2.3571 & 0.74495 & \\
\hline \multirow{2}{*}{ Pair 2 } & 1 month & 2.3571 & 0.74495 & \multirow{2}{*}{0.165} \\
\cline { 2 - 4 } & 2month & 2.0714 & 0.61573 & \\
\hline \multirow{2}{*}{ Pair 3 } & 2month & 2.0714 & 0.61573 & \multirow{2}{*}{0.055} \\
\cline { 2 - 4 } & Third month & 1.7143 & 0.61125 & \\
\hline \multirow{2}{*}{ Pair 4 } & Pretreatment & 3.2143 & 0.69929 & \multirow{2}{*}{$<0.001$} \\
\cline { 2 - 4 } & Third month & 1.7143 & 0.61125 & \\
\hline
\end{tabular}

Table 16.

F-G moderate response, in good response group they had significant differences between pretreatment and third month. 


\begin{tabular}{|l|c|c|c|c|}
\hline \multicolumn{2}{|c|}{} & Mean & Std. Deviation & P value \\
\hline \multirow{2}{*}{ Pair 1 } & Pretreatment & 3.3750 & 0.74402 & \multirow{2}{*}{0.170} \\
\cline { 2 - 4 } & 1 1month & 3.1250 & 0.99103 & \\
\hline \multirow{2}{*}{ Pair 2 } & 1 month & 3.1250 & 0.99103 & \multirow{2}{*}{0.351} \\
\cline { 2 - 4 } & 2month & 3.0000 & 1.19523 & \\
\hline \multirow{2}{*}{ Pair 3 } & 2month & 3.0000 & 1.19523 & \multirow{2}{*}{1} \\
\cline { 2 - 4 } & Third month & 3.0000 & 1.19523 & \\
\hline \multirow{2}{*}{ Pair 4 } & Pretreatment & 3.3750 & 0.74402 & \multirow{2}{*}{0.197} \\
\cline { 2 - 4 } & Third month & 3.0000 & 1.19523 & \\
\hline
\end{tabular}

Table 17.

F-G no response, in non-response group they had no significant differences between pre-treatment and all months.

\begin{tabular}{|l|c|c|c|c|}
\hline & Time & Mean & Std. Deviation & P value \\
\hline \multirow{2}{*}{ Pair 1 } & Pretreatment & 3.0000 & 1.00000 & \multirow{2}{*}{0.423} \\
\cline { 2 - 4 } & 1month & 2.6667 & 0.57735 & \\
\hline \multirow{2}{*}{ Pair 2 } & 1 month & 2.6667 & 0.57735 & \multirow{2}{*}{0.423} \\
\cline { 2 - 4 } & 2 month & 2.3333 & 1.15470 & \\
\hline \multirow{2}{*}{ Pair 3 } & 2month & 2.3333 & 1.15470 & \multirow{2}{*}{0.184} \\
\cline { 2 - 4 } & Third month & 1.6667 & 0.57735 & \\
\hline \multirow{2}{*}{ Pair 4 } & Pretreatment & 3.0000 & 1.00000 & \multirow{2}{*}{0.057} \\
\cline { 2 - 4 } & Third month & 1.6667 & 0.57735 & \\
\hline
\end{tabular}

Table 18.

F-G mild response, in mild response group they had also no significant differences between pretreatment and all months.

\begin{tabular}{|c|c|c|c|}
\hline & Mean & Std. Deviation & P value \\
\cline { 1 - 3 } Pre-treatment & 9.5263 & 0.55687 & \\
\cline { 1 - 3 } First month & 5.5526 & 2.29803 & \multirow{2}{*}{$<0.001$} \\
\cline { 1 - 3 } Second month & 5.0789 & 2.63430 & \\
\hline Third month & 4.9211 & 2.64454 & \\
\hline
\end{tabular}

Table 19.

Visual analogue score, There was highly significant difference in visual analogue score in different months which is higher pre-treatment and lowest in third month (Friedman test).

\begin{tabular}{|l|l|l|l|}
\hline & Mean & Std. Deviation & P value \\
\cline { 1 - 3 } pretreatment & 9.5385 & 0.51887 & \\
\cline { 1 - 3 } First month & 3.0000 & 1.08012 & \multirow{2}{*}{$<0.001$} \\
\cline { 1 - 3 } Second month & 2.1538 & 0.89872 & \\
\cline { 1 - 3 } Third month & 2.1538 & 0.89872 & \\
\hline
\end{tabular}

Table 20.

Visual analogue-good response, There was significant difference between pre-treatment with all other periods and significant difference between first month and second and third month but no significant difference between second and third month.

\begin{tabular}{|c|c|c|c|}
\hline & Mean & Std. Deviation & P value \\
\cline { 1 - 3 } pretreatment & 9.3571 & 0.63332 & \\
\cline { 1 - 3 } First month & 5.8571 & 0.77033 & \multirow{2}{*}{$<0.001$} \\
\cline { 1 - 3 } Second month & 5.4286 & 1.22250 & \\
\cline { 1 - 3 } Third month & 5.0000 & 1.46760 & \\
\hline
\end{tabular}

\section{Table 21.}

Visual analogue-moderate response, The significant difference is only between pretreatment and other periods while between the first, second, and third months no significant difference.

\begin{tabular}{|c|c|c|c|}
\hline & Mean & Std. Deviation & P value \\
\hline Pretreatment & 9.7500 & 0.46291 & \\
\cline { 1 - 3 } First month & 8.3750 & 1.18773 & \multirow{2}{*}{$<0.001$} \\
\cline { 1 - 3 } Second month & 8.3750 & 1.18773 & \\
\cline { 1 - 3 } Third month & 8.3750 & 0.91613 & \\
\hline
\end{tabular}

Table 22.

Visual analogue-no response, the significant difference is only between pre-treatment and other periods while between the first, second, and third months no significant difference. 


\begin{tabular}{|c|c|c|c|}
\hline & Mean & Std. Deviation & P value \\
\hline Pretreatment & 9.6667 & 0.57735 & \multirow{2}{*}{0.043} \\
\cline { 1 - 3 } First month & 7.6667 & 0.57735 & \\
\cline { 1 - 3 } Second month & 7.3333 & 1.15470 & \\
\cline { 1 - 3 } Third month & 7.3333 & 1.15470 & \\
\hline
\end{tabular}

Table 23.

Visual analogue-mild response, the significant difference is only between pre-treatment and other periods while between the first, second, and third months no significant difference.

\section{Discussion}

Many of antiandrogen drugs such as spironolactone, finasteride, cyproterone acetate, flutamide have been used in the treatment of hirsutism as a single drug or in combination $[8,9]$. Long period of treatment is always required to improve hirsutism and to prevent or to delay its relapse, increased 5 $\alpha$-reductase activity has been shown on the skin of the women with idiopathic hirsutism [10].

The use of finasteride for treatment of hirsutism is logical because of its specific effect on $5 \alpha$-reductase, the enzyme responsible for sensitizing the hair to testosterone. In other studies, finasteride administered orally has been successfully used in the treatment of hirsutism [11, 12]. Its contraindications in women due to its effect on the developing male fetus. It inhibits development of normal male genitalia [11]. In our study we compared pretreatment and post treatment for the color of the hairs and the hair removal frequency and the pain during hair removal for four groups which are good response group and moderate response group and mild response group and no response group and by subjective evaluation of all the patients noted a diminished rate of hair growth (fewer times needed for shaving) and a decrement in the density of hairs on the chin area and change in color of the hair but there was no significant difference between groups regarding hair color and there were highly significant difference in hair removal frequency before and after treatment $(\mathrm{P}<0.001)$ when we used finasteride cream $1 \%$ for 3 months.

For other studies by subjective evaluation, Six months of topically applied finasteride $(0.5 \%)$ does not effect on number and thickness of facial hirsutism significantly. Despite lack of objective changes, on questioning, most patients in finastride group perceived a decrease in hair growth with time. As they taken 25 patients noted a considerable diminished rate of hair growth on both sides of the face, especially on the one side that they had guessed that side is medication. In comparison with baseline, hair counts in both finasteride- applied and placebotreated areas decreased significantly 
$(\mathrm{P}<0.0001)$ and respectively. The hair density also have significant diminished at both sides, as compared finastride and placebo treated groups with together [15], as I agree with the decreasing the hair growth but disagree with lack of the objective changes. For other previous studies, Finasteride cream is an effective and harmless drug in patients with idiopathic hirsutism as they enrolled 40 hirsute idiopathic women, taking finasteride cream $0.25 \%$ twice daily for 6 months on their chins. FerrimanGallwey score of the chin area was measyred and result of them was Mean Ferriman-Gallwey score was decreased from $3.2 \pm 0.41$ to $2.2 \pm 0.76(\mathrm{P}<0.001)$. Acne also appeared by 8 patients $(20 \%)$ during the treatment. And there is no other side effects [16].

Finasteride have good result when administered orally in the treatment of hirsutism [8]. Reported that FerrimanGallwey scores in 14 women with idiopathic hirsutism have significant decreasing during a 1-year course of finasteride therapy. In addition, Faloia and colleagues [17] observed the hirsute patients have $33 \%$ reduction in clinical scores after six months from usage of finasteride. Notably there have been fewer investigations about topical application of finasteride. In fact its effects as a topical drug in the treatment of hirsutism are still debated. Leopoldo Falsetti, et al. 1999 [18] showed that Both antiandrogens (finasteride and flutamide) are effective in the treatment of hirsutism; but flutamide effective significantly more than finasteride and flutamide have no chage in the hormone profile; and also both antiandrogen drugs do not have any change in the menstrual cycles in women with idiopathic hirsutism or its irregularity in women with PCOS; and flutamide also has a high risk/benefit ratio and its chronic administration need a repeated liver function tests. Flutamide reduced the Ferriman-Gallwey score with idiopathic hirsutism by $47.7-56.5 \%$, whereas finasteride reduced the score by 29.6-37.9\% in idiopathic hirsutism.

Keles F 2004 [19] showed that free testosterone levels slightly decreased in women when treated with spironolactone. This confirms that the anti-androgenic effect of spironolactone is primarily related to its peripheral effect. 1 year of treatment with a combination of spironolactone and finasteride will result in significant decreasing in free testosterone levels. In this study the hormone profile of the patients with idiopathic hirsutism present significant difference in level of 
free testosterone in good response group compared with the other groups. While in other study, the hormone profile of the patients with idiopathic hirsutism did not present any significant difference compared with the control group [18].

In the present study, no significant differences in the FSH, LH, and TSH levels were found in either group with agreement (Fahri Bayram 2002) [20].

\section{Competing interests}

Authors declare that we have no competing interests.

\section{Authors Contributions}

All authors wrote, read and approved the final manuscript.

\section{References}

1. Rosenfield RL. Clinical practice. Hirsutism. N Engl J Med 2005; 353: 2578-88.

2. Habif TP. Clinical dermatology: A color guide to diagnosis and therapy, 5th edition, chapter 24, Mosby elsevier inc., china: 926-931, 2010.

3. Al-Robaee A, Al-Zolibani A, AlShobaili A, Aslam M. Update on hirsutism. Acta Dermatoven APA 2008; 17(3):103-119.

4. Knochenhauer ES, Key TJ, KahsarMiller M, Waggoner W, Boots LR, Azziz R. Prevalence of the polycystic ovary syndrome in unselected black and white women of the southeastern United States: A prospective study. Journal Clin Endocrinol Metab 2009; 83:3078-3082.
5. Camacho F, Randall VA, Price VH and eds. Hair and its Disorders. Biology, Pathology and Management. London: Martin Dunitz 2000; 359-67.

6. Kahsar-Miller M, Azziz R. Heritability and the risk of developing androgen excess. J Steroid Biochem Mol Biol 1999; 69:261-268.

7. Castello R, Tosi F, Perrone F et al. Outcome of long-term treatment with the 5 alpha-reductase inhibitor finasteride in idiopathic hirsutism: clinical and hormonal effects during a 1-year course of therapy and 1-year follow-up. Fertil Steril 1996; 66:734740.

8. Bayram F, U“ nlu” hızarcı K, Keles, Timur F. Potential utility of insulin sensitizers in the treatment of patients with polycystic ovary syndrome. Treatments in Endocrinology 2002; 1 45-53.

9. Rittmaster RS. Hirsutism. Lancet 1997; 349:191-195.

10. Sahin Y, Dilber S, Kelestimur F. Comparison of Diane 35 and Diane 35 plus finasteride in the treatment of hirsutism. Fertile steril 2001; 75(3): 496-500.

11. Moghetti P, Tosi F, Tosti A, et al. Comparison of spironolactone, flutamide, and finasteride efficacy in the treatment of hirsutism: a randomized, double blind, placebocontrolled trial. Journal of Clinical Endocrinology and Metabolism 2000; 85:89-94.

12. Mu" derris II, Bayram F, Sahin Y, et al. The efficacy of $250 \mathrm{mg} /$ day flutamide in the treatment of patients with hirsutism. Fertility and Sterility 1996; 66:220-222.

13. Conn JJ, Jacobs HS. The clinical management of hirsutism. European Journal of Endocrinology 1997; 136 339-348. 
14. Iraji F, Karbasioun S, Aminorroaya A. Topical Finasteride in Hirsutism: A Double Blind Randomized Clinical Trial on Adult Women. JRMS 2005; 10(6):337-342.

15. Heydari I, Amiri A, Razmjou S, Seyfodin. The Efficacy of Topical Finasteride in the treatment of idiopathic hirsutism. J Turk Acad Dermatol 2008; 2(2):jtad82201a.

16. Faloia E, Filipponi S, Mancini V, Di Marco S, Mantero F. Effect of finasteride in idiopathic hirsutism. $J$ Endocrinol Invest 1998; 21:694-698.

17. Falsetti L, Gambera A, Legrenzi L, Iacobello C, Bugari G. Comparison of finasteride versus flutamide in the treatment of hirsutism. Eur J Endocrinol 1999; 141(4):361-7.
18. Kelestimur F, Everest H, Unluhizarci K $t$ al. A comparison between spironolactone and spironolactone plus finasteride in the treatment of hirsutism. Eur J Endocrinol 2004; 150:351-4.

19. Bayram F, Müderris II, Guven M, Keles F. Comparison of high-dose finasteride ( $5 \mathrm{mg} /$ day) versus low-dose finasteride ( $2.5 \mathrm{mg} /$ day) in the treatment of hirsutism European Journal of Endocrinology 2002; 147:467-471. 